\title{
Determination of the preferred reaction pathway of acetophenone on Si(001) using photoelectron diffraction
}

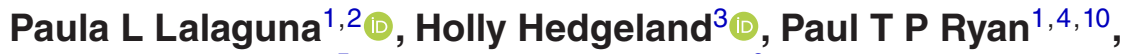 \\ Oliver Warschkow ${ }^{5}$, Matthias K Muntwiler ${ }^{6} \odot$, Andrew V Teplyakov ${ }^{7}$, \\ Steven R Schofield ${ }^{8,9, *}$ and David A Duncan ${ }^{1, *}$ (1)
}

1 Diamond Light Source, Harwell Science and Innovation Campus, Didcot, OX11 0QX, United Kingdom

2 School of Chemistry, University of Glasgow, G12 8QQ, United Kingdom

3 School of Physical Sciences, The Open University, Walton Hall, Milton Keynes, MK7 6AA, United Kingdom

${ }^{4}$ Department of Materials, Imperial College London, SW7 2AZ, United Kingdom

5 Centre for Quantum Computation and Communication Technology, School of Physics, University of Sydney, NSW 2006, Australia

${ }^{6}$ Laboratory for Micro and Nanotechnology, Paul Scherrer Institute, Forschungsstrasse 111, 5232 Villigen PSI, Switzerland

7 Department of Chemistry and Biochemistry, University of Delaware, Newark, DE 19711, United States of America

${ }^{8}$ London Centre for Nanotechnology, University College London, WC1H 0AH, United Kingdom

9 Department of Physics and Astronomy, University College London, WC1E 6BT, United Kingdom

E-mail: david.duncan@diamond.ac.uk and s.schofield@ucl.ac.uk

Received 14 December 2020, revised 12 January 2021

Accepted for publication 16 February 2021

Published 4 May 2021

\begin{abstract}
The adsorption configurations of a technologically relevant model organic adsorbate on the silicon (001) surface were studied using energy scanned $\mathrm{x}$-ray photoelectron diffraction $(\mathrm{PhD})$. Previous work has established the existence of an interesting vertically-aligned ('flagpole') configuration, where the acetophenone attaches to $\mathrm{Si}(001)$ via the acetyl group carbon and oxygen atoms. Density functional theory calculations have predicted two energetically similar variants of this structure, where the phenyl ring is orientated parallel or perpendicular to the rows of silicon dimers on this reconstructed surface. However, previously published experimental measurements, including scanning tunnelling microscopy, $\mathrm{x}$-ray photoelectron spectroscopy, and near-edge $\mathrm{x}$-ray absorption fine structure investigations were unable to distinguish between these two configurations. Here, we apply the unique experimental capabilities of the $\mathrm{PhD}$ technique to this system and demonstrate that the dominant adsorption configuration has the phenyl ring parallel to the dimer rows (the end-bridge structure). This information in turn facilitates the determination of the dominant reaction pathway for acetophenone on $\mathrm{Si}(001)$, which has remained elusive until now. Information about subtle preferences in reaction pathways that affect the alignment and orientation of organic adsorbates such as acetophenone on technologically-relevant semiconductor surfaces such as
\end{abstract}

\footnotetext{
* Authors to whom any correspondence should be addressed.

${ }^{10}$ Current address: Institute of Applied Physics, TU Wien, Vienna, Austria. 
$\mathrm{Si}(001)$ is critical for the fabrication of future atomically-precise atomic and molecular-scale electronic devices utilising the organic-silicon interface, and this work demonstrates the unique and complementary capabilities of $\mathrm{PhD}$ for providing this information.

Keywords: structure determination, organic-silicon interface, Si(001), energy scanned photoelectron diffraction, density functional theory

S Supplementary material for this article is available online

(Some figures may appear in colour only in the online journal)

\section{Introduction}

As fundamental length scale limits are being reached in the utilisation of silicon in the semiconductor industry, the pursuit of novel electronic devices has become a necessity. With a view to shrinking the size of electronic components even more, the organic-silicon interface has drawn significant attention to the development of miniature devices that function on the molecular level [1-6]. However, incorporating molecular components into silicon-based technology presents many challenges. For example, molecules that only bind weakly to the substrate result in structures that are unstable or the silicon surface being incompletely passivated, with the resulting dangling bonds leading to deleterious electronic effects [7]; alternatively, poor electronic overlap between the molecular orbitals and the band structure of the substrate can necessitate high electric fields to align the two, resulting in unwanted dynamic changes to the molecular structure $[1,8]$. Thus, developing methods to robustly attach molecules in precise adsorption configurations with appropriate electronic coupling to the substrate and that are robust under applied electric fields remains a significant challenge.

The ambiphilic nature of the $\operatorname{Si}(001)-(2 \times 1)$ reconstruction involves buckled dimers with a charge transfer of about 0.36 electrons from the down-buckled to the up-buckled dimer atom [9-11]. The electron-poor down-buckled atom presents a strongly reactive site for nucleophiles such as methanol [12] and phosphines [13], while select electrophiles such as borane have been shown to react with the electron-rich up-buckled atom [14]. Molecules containing carbonyl bonds constitute a particularly interesting class of adsorbate species because, during a sequence of reaction steps, they behave both as nucleophiles through the lone pair of the carbonyl oxygen, and electrophiles through the electron-deficient carbonyl carbon [15]. Moreover, acetone and acetophenone have been shown to form a strong $\mathrm{O}-\mathrm{Si}$ bond with the silicon substrate, forming structures that are stable under thermal annealing and within a high local electric field, e.g. between the surface and the tip of a scanning tunnelling microscope (STM) $[16,17]$.

Our prior work using near edge $\mathrm{x}$-ray absorption fine structures (NEXAFS) [18], density functional theory (DFT), and STM $[16,17]$ to study the adsorption of acetophenone on $\mathrm{Si}(100)$ demonstrated that, after room temperature deposition, the phenyl ring of the molecule is found to align mostly parallel to the surface plane with two different orientations (figures 1(a) and (b)). These flat lying molecules were assigned to an allyl structure, where the aromaticity of the phenyl ring is broken by coordination of the two carbon atoms of the phenyl ring to two silicon atoms of the surface. Two distinct orientations of this allyl species were assigned to two different anchoring geometries of the terminal oxygen and carbon atoms of the acetyl group to the surface silicon dimers. Specifically, the terminal atoms bridging two atoms from different silicon dimers (end bridge allyl, EB-allyl, see figure 1(a)), or anchored to two atoms from the same silicon dimer (dimer bridge allyl, DBallyl, see figure 1(b)). We found that the initial allyl adsorption structure could be manipulated into a structure with greater thermodynamic stability: after mild annealing, or via application of a voltage/current pulse from the STM tip, the adsorbate changes configuration such that the phenyl ring is mostly perpendicular to the surface plane, referred to as the flagpole structure. This transition is also deduced from the carbon $1 \mathrm{~s} \mathrm{x}$ ray photoelectron spectroscopy (XPS) data, where a new peak appears at lower binding energy due to hydrogen dissociation from the methyl group [18]. This flagpole structure is both thermodynamically stable, and exhibits strong electronic coupling to the silicon substrate $[16,18]$, making this adsorbate desirable for device applications, or as an anchoring group for further functionalisation.

As with the allyl structure, two orientations of the flagpole structure with respect to the surface silicon dimers are possible: bridging across two Si dimers (end bridge flagpole, EBflagpole, see figure 1(c)), or bridging a single Si dimer (dimer bridge flagpole, DB-flagpole, see figure 1(d)). Knowledge of the orientation of this flagpole structure is important in the context of molecular device fabrication for two reasons: (1) it provides a means to determine experimentally the preferred reaction pathway on the silicon surface, which is important not only for this adsorbate, but the class of adsorbates that anchor to silicon via an acetyl group; and (2) the orientation of the adsorbate with respect to the surface dimers can be a critical factor in the further functionalisation of the surface [19]. While STM is capable of differentiating between the two orientations of the allyl features (figures 1(a) and (b)), the large aspect ratio of the two flagpole structures (figures 1(c) and (d)) makes differentiation between these two features impossible using STM. NEXAFS is similarly incapable of determining 
a) End-bridge allyl

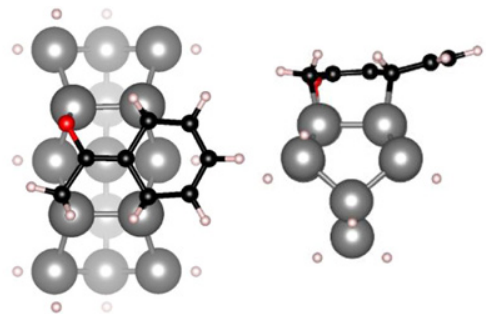

c) End-bridge flagpole
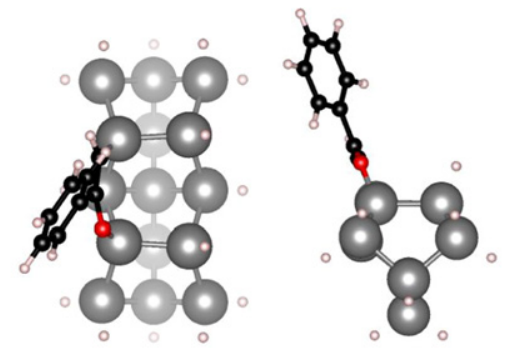

e) Molecular adsorption
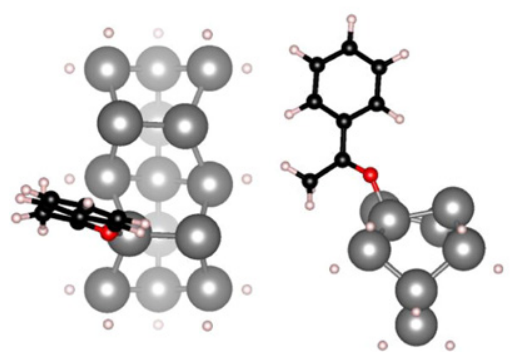

b) Dimer-bridge allyl

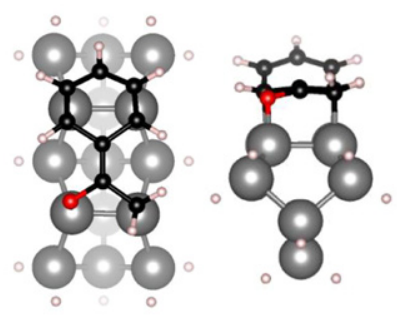

d) Dimer-bridge flagpole

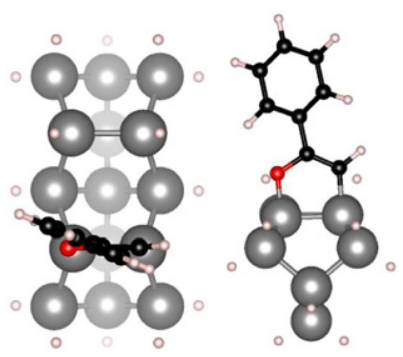

f) $2+2$ cycloaddition $\mathrm{C}=\mathrm{O}$

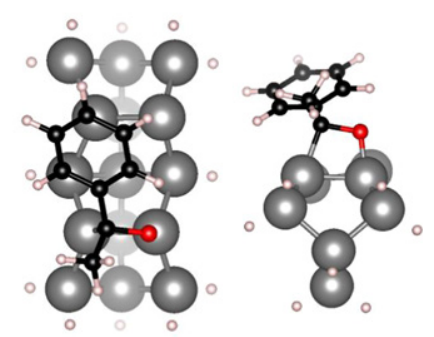

Figure 1. Top and side views of the structures predicted by DFT: (a) EB-allyl, (b) DB-allyl, (c) EB-flagpole, (d) DB-flagpole, (e) molecular adsorption, (f) $2+2$ cycloaddition $\mathrm{C}=\mathrm{O}$. The black atoms are $\mathrm{C}$, white $\mathrm{H}$, red $\mathrm{O}$ and grey $\mathrm{Si}$. In the plan views the $\mathrm{Si}$ atoms below the dimers are shaded in a lighter colour as a function of depth.

the azimuthal orientation of the molecule and thus differentiating the two models [20] due to the four fold symmetry of the surface, nor can these structures be differentiated using XPS.

For completeness, in this work we also consider two additional structures that were not part of earlier DFT calculations. These are the molecularly adsorbed configuration, shown in figure $1(\mathrm{e})$ and a $\mathrm{C}=\mathrm{O} 2+2$ cycloaddition structure shown in figure 1(f). These structures were modelled, since we considered it plausible that they might produce a fit to the photoelectron diffraction $(\mathrm{PhD})$ data; however, as described below, this did not turn out to be the case and these structures were ruled out.

Energy-scanned $\mathrm{x}$-ray $\mathrm{PhD}$ is arguably the only technique with the potential for experimentally determining the structural properties of the acetophenone flagpole structure on $\mathrm{Si}(001)$. This technique exploits the coherent interference of the directly-emitted photoelectron component, and components of the same photoelectron wavefield elastically backscattered by neighbouring atoms [21]. As the photon energy, and therefore the photoelectron kinetic energy/photoelectron wavelength, is varied, different scattering pathways come into and out of phase, yielding modulations in the photoemission intensity. These modulations contain information about the relative distances and orientation between the emitter and the scatterers, and allow us to obtain precise structural information without requiring long range order.

In this work, we explore the powerful and unique capabilities of $\mathrm{PhD}$ for determining detailed structural information for a model adsorbate (acetophenone) adsorbed on the technologically ubiquitous silicon (001) surface. In particular, we measure silicon (001) surfaces exposed to acetophenone before and after being subjected to mild thermal annealing. Using this method, we determine the preferred orientation of the acetophenone flagpole structure, thereby elucidating the preferred kinetic pathway of the acetophenone adsorbate among those that have been theoretically predicted $[16,17]$. We also discuss the limitations of this technique for the precise determination of structural parameters of organic adsorbates on silicon. 


\section{Experimental and computational details}

\subsection{Experimental details}

The experiment was performed at the PEARL beamline [22] of the Swiss Light Source. The end-station of the PEARL beam line consists of an analysis chamber, a low-temperature STM chamber and a preparation chamber. All chambers have a base pressure $\sim 3 \times 10^{-10}$ mbar. A clean $\operatorname{Si}(001)$ (As-doped, $0.0015-0.0400 \Omega \mathrm{cm}$, Virginia Semiconductor) surface was prepared by repeated resistive annealing of the silicon crystal with $45 \mathrm{~W}(6 \mathrm{~A} / 7.5 \mathrm{~V})$ for $20 \mathrm{~s}$ with $1 \mathrm{~min}$ ramp down from $2 \mathrm{~A}$, with the chamber pressure staying below $\sim 5 \times$ $10^{-9}$ mbar. The crystalline quality of the surface was judged by STM measurements; the cleanliness of the surface by synchrotron XPS (SXPS). Acetophenone was exposed to the sample through a leak valve at a partial pressure of $1 \times 10^{-8} \mathrm{mbar}$ for $300 \mathrm{~s}\left(\sim 3 \mathrm{~L}\right.$, where $1 \mathrm{~L}$ is $1 \times 10^{-6}$ mbar s $)$. Prior to exposure the gas line between the acetophenone container and the leak valve was baked and then conditioned under a flow of acetophenone. Prior to annealing the acetophenone layer, the sample was re-prepared with a new acetophenone layer in order to avoid effects from potential beam damage. To anneal the acetophenone layer, the $\mathrm{Si}$ sample was again resistively annealed but at a lower power of $1.11 \mathrm{~W}(3.7 \mathrm{~V} / 0.3 \mathrm{~A})$ for $10 \mathrm{~min}$. Oxygen 1s, carbon 1s and silicon 2p SXPS measurements were taken of both preparations [shown in the ESI, figure S1 (https://stacks.iop.org/JPCM/33/214002/mmedia)], and conform well to our previously published results [18].

The XPS and PhD measurements were acquired from a Scienta EW4000 hemispherical electron energy analyser. The analyser was mounted at an angle of $\sim 60^{\circ}$ with respect to the incident beam in the plane of the photon polarisation (linear horizontal). The slits of the detector were orientated perpendicular to polarisation of the incident light and, as such, for the $\mathrm{PhD}$ measurements it was considered as a point detector utilising only the inner $\pm 5^{\circ}$ of the analyser's acceptable angle. The end-station is equipped with a six-axis manipulator, allowing translations in $x, y$ and $z$ and polar, azimuthal and tilt rotations.

Oxygen 1s PhD modulation spectra were recorded for both preparations in the photon energy range of $\sim 630-890 \mathrm{eV}$, with an energy step of $4 \mathrm{eV}$. The data were acquired along the [110] and [100] crystallographic directions in the polar emission angle range of $0^{\circ}-60^{\circ}$. The resulting energy distribution curves were processed using our standard methodology [21, 23], whose details can be found in the ESI. Carbon $1 \mathrm{~s} \mathrm{PhD}$ modulation spectra were also acquired (see figure S2 in the ESI), but no significant modulations were observed, thus fitting of this data was not pursued and the quantitative analyses were based solely on the $\mathrm{O} 1 \mathrm{~s} \mathrm{PhD}$ modulations. A full list of the fitting parameters that were allowed to vary in the calculations is detailed in the ESI. The three fitting parameters that were found to strongly affect the fitting were those defining the bond vector between the oxygen atom and the silicon atom it was bound to. These positions were defined in spherical coordinates: bond length $(R)$, tilt of the bond away from the surface normal $(\theta)$ and the rotation of the bond away from the [110] direction $(\phi)$. The other fitting parameters are not discussed as, individually, they had negligible effect on the quality of the fit.

To compare the agreement between the resulting $\mathrm{PhD}$ and the DFT structures a fitness parameter, $\Delta F$, was introduced:

$$
\begin{gathered}
\Delta F=\sqrt{\sum_{i} F_{(i)}^{2}}, \\
F_{i}=\frac{X_{(i)}^{\mathrm{PhD}}-X_{(i)}^{\mathrm{DFT}}}{\sigma_{(i)}} .
\end{gathered}
$$

Here $X_{(i)}^{\mathrm{PhD}}$ and $X_{(i)}^{\mathrm{DFT}}$ are the values of structural parameter $i$ of the $\mathrm{PhD}$ and DFT structures, respectively; $\sigma_{i}$, is the associated uncertainty in that fitting parameter from the $\mathrm{PhD}$ structure with one of the best found $R$-factor, $R_{\mathrm{fac}}$. The three structural parameters used for this calculation were $R, \theta$ and $\phi$, with associated uncertainties of $0.05 \AA, 4^{\circ}$ and $12^{\circ}$, respectively. Any structure with a value of $\Delta F$ greater than 2 is considered significantly different from the DFT model.

\subsection{Computation details}

DFT calculations were performed using the Gaussian 09 suite of programs [24]. The surface was modelled by a $\mathrm{Si}_{15} \mathrm{H}_{16}$ cluster that represented two silicon dimers within the same row. Calculations were performed using the B3LYP method [25, 26] with the 6-311 $+G(d, p)$ basis set paired with Grimme's D3 dispersion corrections [27] and original damping function. The coordinates of atoms representing third through fifth rows of silicon atoms in the model clusters were fixed at bulk positions to avoid unrealistic distortion. After the $\mathrm{Si}_{15} \mathrm{H}_{16}$ cluster was optimized, the appropriate adsorbate was placed on the sites representing the surface and the structure was reoptimized at the same level of theory and with the same geometrical constraints. Adsorption energies were calculated from total energy calculations according to the formula:

$$
\begin{aligned}
E_{\text {adsorption }}= & E_{\text {adsorbate }+ \text { substrate }} \\
& -\left(E_{\text {substrate }}+E_{\text {gas phase molecule }}\right) .
\end{aligned}
$$

In the case of the two allyl structures, adsorption of the molecule to the surface is dissociative, resulting in the formation of a surface silicon hemihydride dimer. In these two cases, the adsorption energy was calculated using:

$$
\begin{aligned}
E_{\text {adsorption }}= & E_{\text {adsorbate }+ \text { substrate }}+E_{\text {hemihydride }} \\
& -\left(2 E_{\text {substrate }}+E_{\text {gas phase molecule }}\right) .
\end{aligned}
$$

\section{Results}

\subsection{DFT modelling}

Six unique structures were modelled in this study. Three of the models are those presented in our prior work [16, 18]: EB-allyl (figure 1(a)), DB-allyl (figure 1(b)) and DB-flagpole (figure 1(d)). We also present calculations of the EB-flagpole (figure 1(c)), in addition to two wholly novel structures: a molecularly adsorbed structure (figure 1(e)) and a $2+2$ cycloaddition structure (figure 1(f)). These two novel 
Table 1. Calculated adsorption energies for the structures shown in figure 1.

\begin{tabular}{ccccccc}
\hline & Molecular & $2+2 \mathrm{C}=\mathrm{O}$ & EB-allyl & DB-allyl & EB-flagpole & DB-flagpole \\
\hline Energy $(\mathrm{eV})$ & -1.12 & -2.02 & -3.39 & -3.27 & -3.76 & -4.15 \\
\hline
\end{tabular}

a)

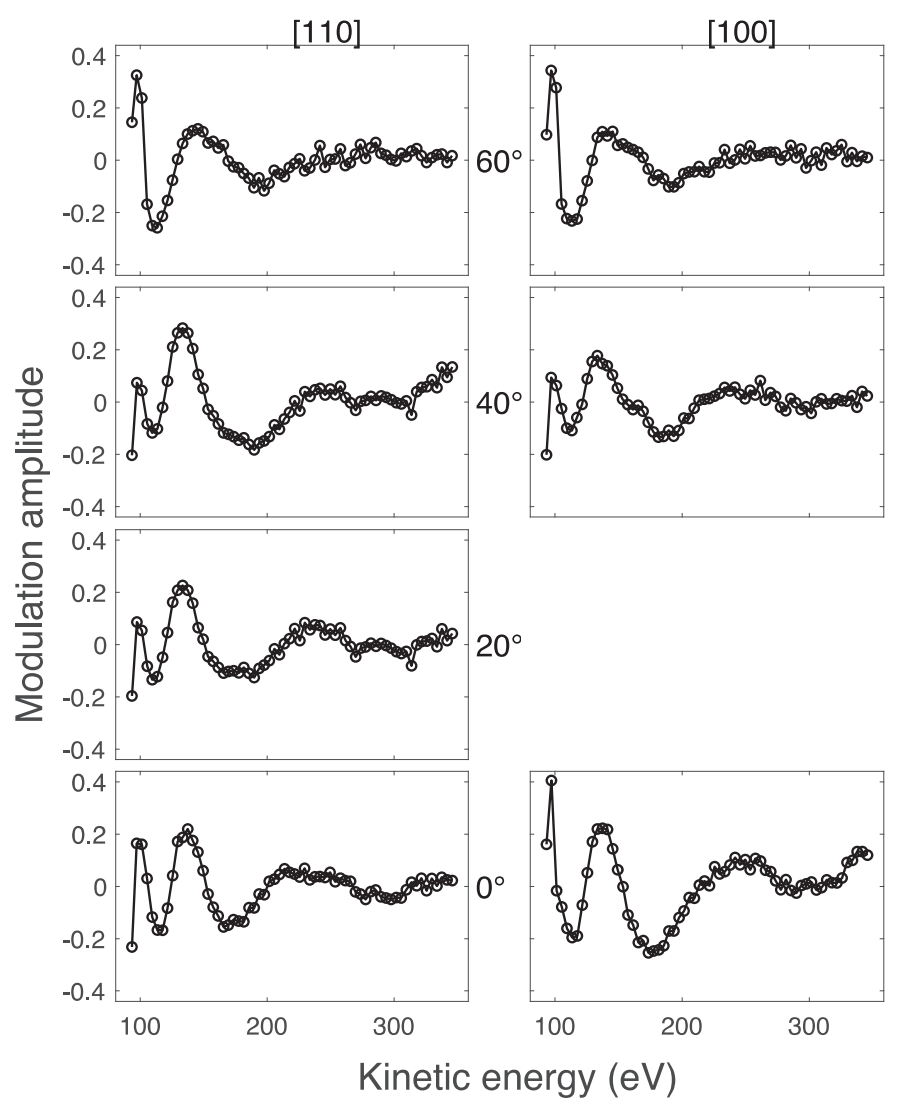

b)

[110]
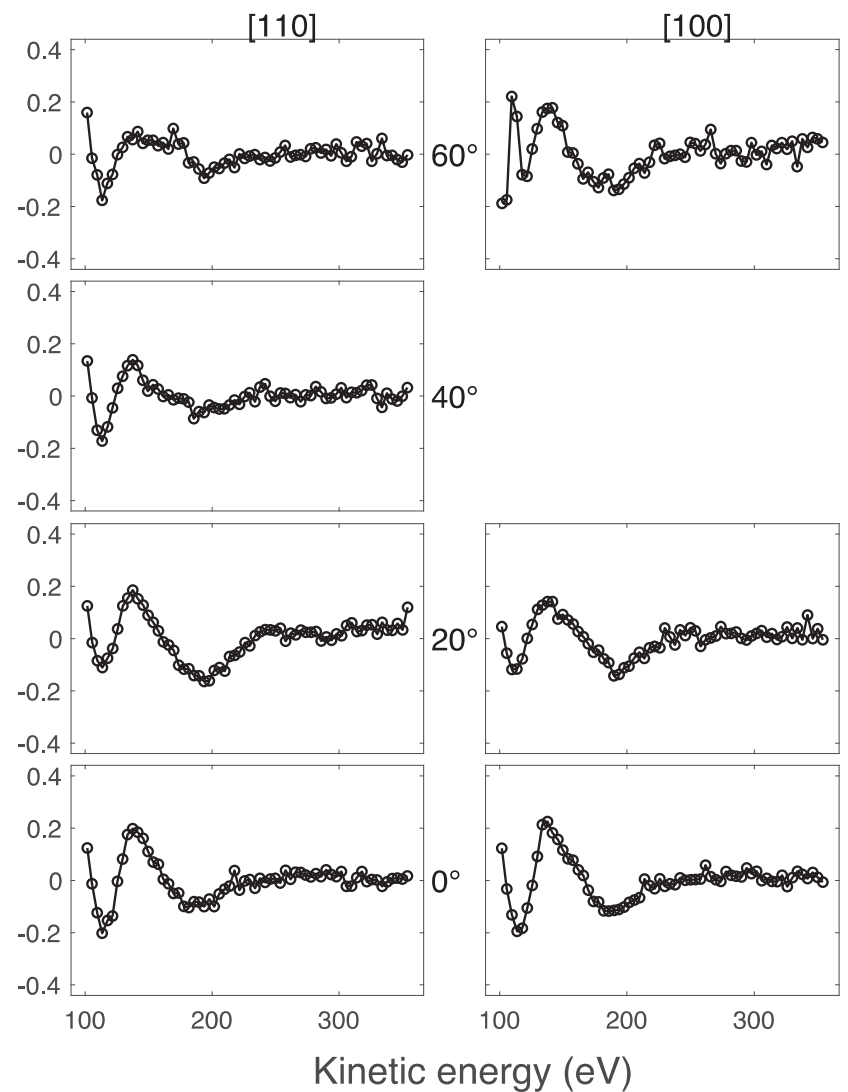

Figure 2. Oxygen 1s modulations amplitudes for (a) the room-temperature and (b) annealed preparation. The azimuth is shown in each column and polar emission angles with respect to normal emission in each row.

structures were found to be significantly less energetically favourable than the allyl and flagpole structures (see table 1). Notably the energy minimised molecular adsorption structure is orientated almost perpendicular to the surface, tilted $83^{\circ}$ away from the surface plane, which would exclude it from being the primary structure present after deposition at room temperature [16, 17]. Furthermore, the methyl group is not deprotonated in this structure, hence it would not account for the appearance of the lower binding energy peak in the C 1 s XPS spectrum after annealing. The $2+2$ cycloaddition structure has the carbon and oxygen atom of the carbonyl bound to two separate silicon atoms. This configuration has the phenyl ring almost parallel to the substrate, tilted $26^{\circ}$ away from the surface plane, which would be consistent with the room temperature deposition phases; however, it is unclear how such a configuration would result in the double lobed structure observed in STM, and is over $1 \mathrm{eV}$ less favourable than either allyl structure. Regardless of this apparent predisposition towards the allyl and flagpole structures, the molecular adsorption and the $2+2$ cycloaddition structures were also considered in the $\mathrm{PhD}$ analysis.

The two allyl structures (end bridge and dimer bridge; EB and DB) have the terminal carbon atom of the acetyl group singly deprotonated; while the two flagpole structures (also EB and DB) have the terminal carbon atom doubly deprotonated. Consistent with prior work, we find here that the flagpole structures are overall more stable than the allyl structures, and the DB-flagpole model is predicted to be the most energetically favourable, with an adsorption energy $0.39 \mathrm{eV}$ lower than that of the EB-flagpole (table 1). The DB- and EB-allyl have a far more comparable adsorption energy, only differing by $0.12 \mathrm{eV}$. The EB- and DB-allyl structures have the phenyl ring orientated more parallel to the substrate, tilted $15^{\circ}$ and $33^{\circ}$ away from the surface plane, respectively. The EB- and DB-flagpole structures, instead, have the phenyl ring almost perpendicular to the substrate, tilted $65^{\circ}$ and $77^{\circ}$ away from the surface plane. Note that in our prior NEXAFS study [18], we showed that at 
Table 2. Best fitting PhD structural models for single site occupation of a given DFT model for the PhD data taken after room-temperature deposition. The structural parameters of the $\mathrm{PhD}$ fit are given, the corresponding parameters from the DFT calculations are shown in brackets.

\begin{tabular}{lccccc}
\hline & $R(\AA)$ & $\phi\left(^{\circ}\right)$ & $\theta\left(^{\circ}\right)$ & $R$-factor & $\Delta F$ \\
\hline EB-allyl & $1.65 \pm 0.06(1.75)$ & $55 \pm 15(66)$ & $19 \pm 4(20)$ & 0.45 & 2.2 \\
DB-allyl & $1.65 \pm 0.05(1.74)$ & $83 \pm 18(83)$ & $15 \pm 5(14)$ & 0.46 & 1.9 \\
EB-flagpole & $1.68 \pm 0.06(1.71)$ & $39 \pm 19(39)$ & $23 \pm 4(26)$ & 0.44 & 1.0 \\
DB-flagpole & $1.63 \pm 0.05(1.72)$ & $16^{\mathrm{a}}(10)$ & $12 \pm 7(5)$ & 0.50 & 2.5 \\
M. adsorption & $1.85 \pm 0.09(1.85)$ & $-5 \pm 11(3)$ & $34 \pm 4(23)$ & 0.61 & 2.9 \\
$2+2$ CO & $1.63 \pm 0.05(1.72)$ & $97 \pm 14(109)$ & $21 \pm 5(9)$ & 0.43 & 3.5 \\
\hline
\end{tabular}

${ }^{\text {a }}$ Varying this parameter across all possible values did not result in an $R$-factor greater than the variance.

Table 3. Best fitting PhD structural models for single site occupation of a given DFT model for the $\mathrm{PhD}$ data taken after annealing the sample. The structural parameters of the $\mathrm{PhD}$ fit are given, the corresponding parameters from the DFT calculation are shown in brackets.

\begin{tabular}{lccccc}
\hline & $R(\AA)$ & $\phi\left(^{\circ}\right)$ & $\theta\left(^{\circ}\right)$ & $R$-factor & $\Delta F$ \\
\hline EB-allyl & $1.65 \pm 0.05(1.75)$ & $57 \pm 27(66)$ & $26 \pm 4(20)$ & 0.40 & 2.6 \\
DB-allyl & $1.65 \pm 0.04(1.74)$ & $82 \pm 11(83)$ & $20 \pm 4(14)$ & 0.44 & 2.4 \\
EB-flagpole & $1.69 \pm 0.05(1.71)$ & $40 \pm 10(39)$ & $31 \pm 3(26)$ & 0.37 & 1.3 \\
DB-flagpole & $1.60 \pm 0.04(1.72)$ & $8^{\mathrm{a}}(10)$ & $3 \pm 8(5)$ & 0.33 & 2.5 \\
M. adsorption & $1.83 \pm 0.16(1.85)$ & $3 \pm 13(3)$ & $34 \pm 5(23)$ & 0.52 & 2.8 \\
$2+2$ CO & $1.62 \pm 0.04(1.72)$ & $114 \pm 9(109)$ & $20 \pm 3(9)$ & 0.31 & 3.3 \\
\hline
\end{tabular}

${ }^{a}$ Varying this parameter across all possible values did not result in an $R$-factor greater than the variance.

room temperature there remains a kinetic barrier to the formation of the flagpole structures that can be overcome by mild thermal annealing.

\subsection{Qualitative analysis of PhD data}

The oxygen $1 \mathrm{~s} \mathrm{PhD}$ data of acetophenone on $\mathrm{Si}(001)$ after room temperature deposition and after annealing the sample are shown in figures 2(a) and (b), respectively. Before performing a quantitative analysis it is useful to gain some insight from a qualitative inspection of the modulations. In particular, when the angle between the emitter, nearest-neighbour scatterer and detector is $180^{\circ}$, the modulation intensity will be at its greatest. Thus, if the emitter atom is adsorbed in a direct atop site (when an atom is directly above another, with no lateral displacement), the strongest modulations will be observed at normal emission. However, if the atom is offatop, weaker modulations will be observed at normal emission, with stronger modulations at higher emission angles. Secondly, short emitter to nearest-neighbour scatterer distances result in long period modulations; long emitter to nearestneighbour scatterer distances result in short period modulations. The $\mathrm{O} 1 \mathrm{~s}$ modulations after room temperature deposition and after annealing (figure 2) are comparably weak ( $\sim \pm 20 \%$ ), and show the strongest modulations at an emission angle between $0^{\circ}$ and $20^{\circ}$ (with respect to normal emission). This suggests that the oxygen atoms are adsorbed in an off-atop site or sites. After annealing, the modulations still exhibit a relatively long period, again suggesting a comparably short oxygen-silicon bond length $(<2 \AA)$. Inspection of the most energetically favourable DFT calculated structures (figure 1) reveals that each of the computed structures has oxygen positioned in an off-atop site, in agreement with this qualitative inspection of the $\mathrm{PhD}$ data. Specifically, the oxygen atom in the DB-allyl is found $0.42 \AA$ off-atop, EBallyl $0.60 \AA$, DB-flagpole $0.16 \AA$, EB-flagpole $0.74 \AA$ off-atop, molecular adsorption $0.71 \AA$ off-atop and $2+2$ CO $0.27 \AA$ offatop. Thus, the EB models adsorb with the oxygen atom more off-atop than the DB models, with EB-flagpole the furthest off-atop.

\subsection{Quantitative PhD: multiple-scattering calculations}

3.3.1. Room-temperature deposited. The best fits determined by multiple-scattering calculations of the six DFT structures as a single adsorption site are shown in table 2, with the corresponding values predicted by DFT shown in brackets. None of these models resulted in an $R$-factor less than 0.44 , indicating poor agreement between the experimental and theoretical modulations in $\mathrm{PhD}$. Furthermore, the agreement between the DFT and $\mathrm{PhD}$ structural parameters is poor for all models $(\Delta F>2)$ except for EB-flagpole $(\Delta F=1.0)$. This indicates that none of the structures in figure 1(a)-(f) on their own can account for the modulations observed in the $\mathrm{PhD}$ data. This is not particularly surprising, since previous STM work $[16,17]$ has already indicated that the surface should be at least a mixture of the two allyl features.

Therefore, we have also performed $\mathrm{PhD}$ modelling considering a surface composed of a various mixture of two out of the six configurations in figures 1(a)-(f). The results of these fittings can be found in the supplementary information (see table $\mathrm{S} 1$ in the ESI). Unfortunately, these two-site models resulted 
a) EB flagpole
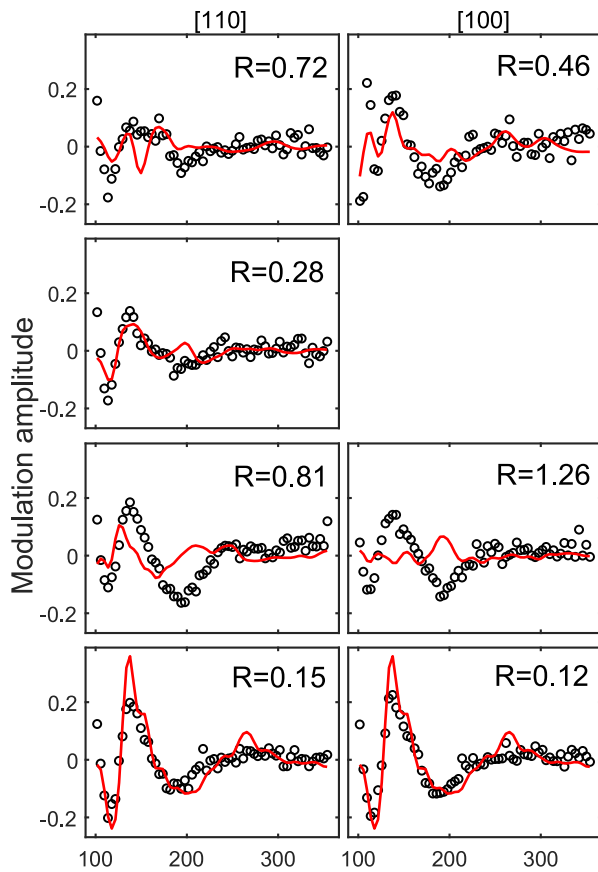

bB flagpole

b) + EB allyl
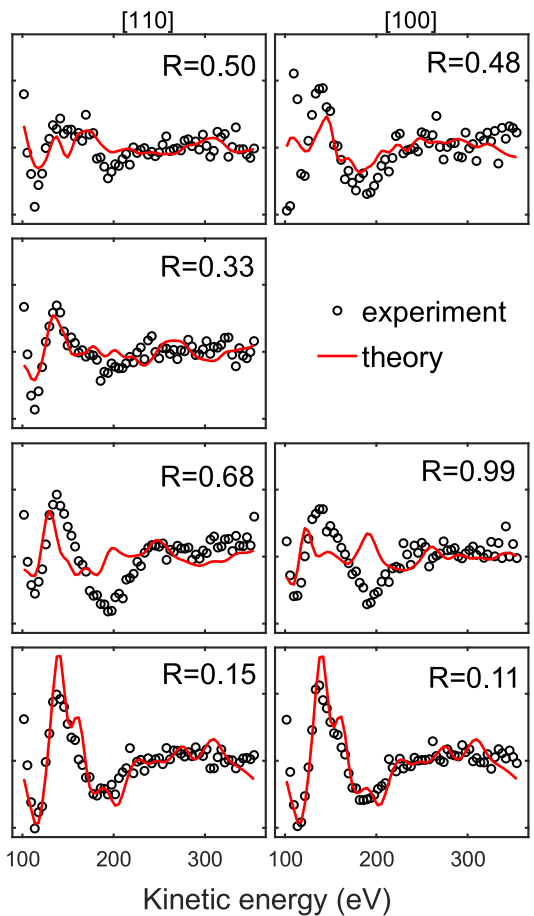

EB flagpole + DB allyl
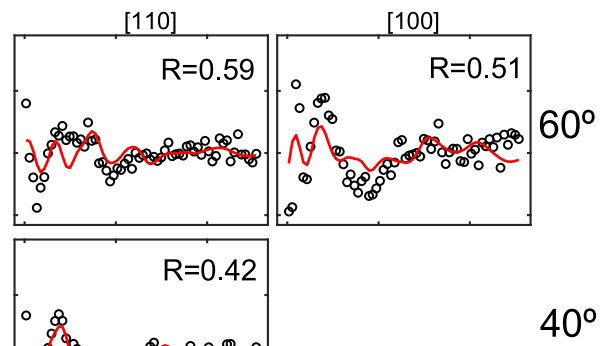

Figure 3. Comparison of the experimental and theoretical $\mathrm{PhD}$ modulations for the best fits found in the annealed preparation. Angles listed on the right-hand side are photoelectron emission angles with respect to normal emission. Inset is the individual $R$-factor for fit of each experimental $\mathrm{PhD}$ spectra to its corresponding theoretical spectra.

in only minor improvements in the $R$-factor (e.g. 0.43 for EBallyl combined with EB-flagpole) and poor agreement of the structural parameters $(\Delta F>2)$. The best $R$-factor (0.39) is observed when EB-flagpole is combined with the $2+2 \mathrm{CO}$ structure, but the agreement with DFT is poor $(\Delta F=3)$. The poor agreement, especially for the two allyl model, at first does seem surprising, since STM observations of the room temperature dosed surface show very clearly a surface composed primarily of two separate allyl features $[16,17]$. However, resolution to this apparent discrepancy comes when the nature of the sample preparation is taken into account. In the STM experiments the surface is exposed to a very low dose of acetophenone in order to clearly identify isolated adsorbates. In contrast, in order to have a sufficient signal-to-noise ratio for the $\mathrm{PhD}$ experiments, the surface must be exposed to a higher dose of acetophenone. In performing our experiments, we observed the XPS signal from the dosed surface and we were careful to keep the dose sub-saturation; nevertheless, the higher coverage apparently results in sufficient percentage of additional configurations as to make accurate determination of the adsorbate structures by $\mathrm{PhD}$ fitting analysis prohibitive, and indeed prior studies have indicated that adsorption at high coverages results in the formation of multiple different local adsorption sites [18, 19].

Thus, we were not able to determine a good fit to confirm the adsorption structures of the room temperature dosed system; both single and two site models failed to adequately explain the experimental $\mathrm{PhD}$ data. Due to the 'trial and error' approach necessary for performing PhD data analysis, it is not realistic to model three or more differing structures, as adding in an ever greater number of fitting parameters is more likely to result in a greater number of non-unique solutions. Nevertheless, it is known that metastable structures, such as the $2+2$ product, can be converted to the more stable structures by thermal anneal or STM activation [15], and as the average tilt angle of the molecule increases with thermal annealing, more space on the surface becomes available to convert these metastable structures into the more stable allyl or flagpoles structures. This suggests that mildly annealing the surface may increase the order and therefore also our chances of obtaining reliable fits to the $\mathrm{PhD}$ data.

3.3.2. Annealed. In good agreement with prior work, the single site allyl models result in both a poor $R$-factor $(>0.4)$ and $\Delta F(>2)$ when used to fit to the annealed surface data. The best fits found for a single-site modelling of the DFT structures by multiple-scattering calculations are shown in table 3 , and the corresponding predicted values by DFT for these parameters are shown in brackets. The best $R$-factors, 0.31 and 0.33 , are found for the $2+2 \mathrm{CO}$ and DB flagpole structures, respectively. However, the bond length in these structures (1.62 \pm $0.04 \AA$ and $1.60 \pm 0.04 \AA$ for $2+2$ CO and DB-flagpole, respectively) is significantly shorter than that predicted by DFT (1.72 A). Notably, the same models with a bond length of $1.72 \AA$ have an $R$-factor of $\sim 1.0$. As such, despite the good $R$-factor, neither the DB-flagpole nor the $2+2 \mathrm{CO}$ structure 
Table 4. Best fitting PhD structural models for two site occupation of a given DFT model for the PhD data taken after annealing the sample. The main structural parameters of the $\mathrm{PhD}$ fit are given, the corresponding parameters from the DFT calculation are shown in brackets.

\begin{tabular}{|c|c|c|c|c|c|c|}
\hline & $R(\AA)$ & $\phi\left(^{\circ}\right)$ & $\theta\left(^{\circ}\right)$ & $\%$ occupation & $R$-factor & $\Delta F$ \\
\hline \multirow[t]{2}{*}{ EB-allyl + EB-flagpole } & $1.7 \pm 0.2(1.75)$ & $70^{\mathrm{a}}(66)$ & $19^{\mathrm{a}}(20)$ & 49 & \multirow[t]{2}{*}{0.35} & \multirow[t]{2}{*}{1.9} \\
\hline & $1.69 \pm 0.04(1.71)$ & $40 \pm 12(39)$ & $33 \pm 3(26)$ & 51 & & \\
\hline \multirow[t]{2}{*}{ DB-allyl + EB-flagpole } & $1.72^{\mathrm{a}}(1.74)$ & $80^{\mathrm{a}}(83)$ & $10^{\mathrm{a}}(14)$ & 14 & \multirow[t]{2}{*}{0.38} & \multirow[t]{2}{*}{1.9} \\
\hline & $1.69 \pm 0.05$ & $38 \pm 12(39)$ & $32 \pm 4(26)$ & 86 & & \\
\hline
\end{tabular}

${ }^{a}$ Varying this parameter across all possible values did not result in an $R$-factor greater than the variance.

provide a good fit to our $\mathrm{PhD}$ data. The next best $R$-factor for our single site modelling is the EB-flagpole, with an $R$-factor of 0.37 . In addition to an excellent $R$-factor, this configuration also has an excellent agreement between the PhD and DFT structural parameters $(\Delta F=1.3)$. The comparison between the experimental and theoretical $\mathrm{PhD}$ modulations is shown in figure 3(a).

Two-site modelling of the annealed system led to only one structure with a better $R$-factor than the single site EB-flagpole structure (see table S2). Specifically, a mixture of EB-allyl and EB-flagpole $\left(R_{\mathrm{fac}}=0.35, \Delta F=1.9\right)$. Only one other two site model resulted in a $\Delta F<2$, which was a mixture of an DBallyl and EB-flagpole $\left(R_{\mathrm{fac}}=0.38\right)$. The comparison between the experimental and theoretical $\mathrm{PhD}$ modulations for both two site models are shown in figures 3(b) and (c) (respectively), and the structural parameters for the fits are shown in table 4 .

\section{Discussion}

$\mathrm{PhD}$ is a powerful tool for the elucidation of atomic-scale structural parameters that are inaccessible to other techniques. However, it is most successful when applied to surfaces with a small number of distinct adsorption configurations. Thus, when applied to our room-temperature dosed surface, where multiple adsorption configurations exist, we were not able to obtain useful fits to our $\mathrm{PhD}$ data.

By annealing the surface, we were able to reduce the number of adsorbate structures, and fitting our $\mathrm{PhD}$ data to a two-species model produced an excellent fit to our data; in particular, the $\mathrm{PhD}$ data clearly indicates that the surface consists of a mixture of the EB-flagpole, and the EB-allyl adsorbate. Thus, our $\mathrm{PhD}$ data resolves the uncertainty that remained after our prior detailed XPS, NEXAFS, STM, and DFT studies [16-18], and identifies the EB-flagpole, and not the DBflagpole, as the predominant structure formed by acetophenone on $\mathrm{Si}(001)$ after room temperature dosing and mild thermal annealing. It is worth noting that this is not the structure that might be anticipated by a naive interpretation of the DFT calculated structures, since the EB-flagpole is predicted to be less thermodynamically stable than the DB-flagpole. This can be attributed to the reaction pathway, from the allyl structures to the flagpole structures, being kinetically controlled.

It is important to note that, although it was possible to achieve a good fit to the combined data set, in none of the structures was a good fit to the $20^{\circ}$ emission angle data found. In figure 3 , the $R$-factors for each individual emission angle are shown, and universally the $20^{\circ}$ emission angle has a notably poor fit $(>0.5)$. While the $40^{\circ}$ and $60^{\circ}$ emission angle data in some of the models also have a numerically poor fit, the comparatively small modulations $(\sim 10 \%)$ found at these emission angles will result in an underestimate in the quality of the fit at these emission angles. However the data at $20^{\circ}$ are almost as strongly modulating as those at normal emission. This suggests that there is an aspect of the adsorbate structure that is not being well modelled within the structures that we have trialled. This could be indicative of a surface reconstruction, or the presence of a minority species on the surface that we have not modelled in our calculations. It is therefore important to qualify what this study was. Within this study we were explicitly comparing how well the theoretically predicted DFT structures match the experimentally measured structural data. Within this constraint, it is clear that the EB flagpole structure provides the best available fit to the experimental data, and thus likely dominates the surface post annealing.

The dominant structure upon room-temperature adsorption is the DB-allyl [16]. To convert the adsorbate from this allyl structure to the flagpole structure requires removing a $\mathrm{H}$ atom from the terminal $\mathrm{C}$ atoms of the acetyl group. There are two possible reaction pathways, one where the hydrogen abstraction occurs directly from the DB-allyl, and the other where the DB-allyl first converts to the EB-allyl [16]. Our results confirm that the latter is the kinetically preferred pathway. Indeed, direct manipulation of the DB-allyl using voltage pulses delivered by an STM tip has demonstrated the ability to induce the DB-allyl to convert to the EB-allyl, but the reverse process was never observed [16]. In addition, we note that the EB-allyl and EB-flagpole structures are found in DFT calculations to have the oxygen and carbon atoms of their acetyl groups in significantly more off-atop positions than the corresponding dimerbridge structures, which is consistent with our observation of weak O 1s modulations. We speculate that the physical proximity of the terminal carbon of the acetyl group in relation to the neighbouring silicon atoms may result in a lowering of the activation energy for the $\mathrm{H}$ abstraction from the EB-allyl over the competing process of $\mathrm{H}$ abstraction from the DB-allyl, contributing to the preference for the reaction pathway resulting in the EB-flagpole structure.

\section{Conclusions}

We have presented an $\mathrm{O}$ 1s energy-scanned $\mathrm{PhD}$ study, with supporting DFT calculations, of the adsorption structures of acetophenone on the technologically pervasive silicon (001) surface. The unique capabilities of the $\mathrm{PhD}$ technique allow 
us to answer a fundamental question regarding the physics and chemistry of this interaction that has remained elusive despite significant prior work with STM, DFT, XPS, and NEXAFS. In particular, we demonstrated that the most likely configuration adopted by the acetophenone adsorbate after room temperature dosing followed by a mild thermal anneal is an end-bridge flagpole configuration. This is despite DFT calculations suggesting that a 90 degree azimuthally-rotated structure, the dimer-bridge flagpole, is energetically favoured. Our results indicate that the surface reaction is kineticallylimited, and allow us to identify the preferred reaction pathway, where the acetophenone adsorbate transitions through the end-bridge allyl configuration. We hasten to add that this work has focussed on contrasting specific models predicted by DFT against our experimental $\mathrm{PhD}$ measurements, however both DFT and $\mathrm{PhD}$ are inherently imagination limited: if the basic structural parameters of the real structure are not present in our starting models, the real structure will not be found. With that limitation in mind, as interest in molecular adsorption on semiconductors progresses from small uni-functional adsorbates, to larger, technologically-relevant multi-functional adsorbates, we anticipate that $\mathrm{PhD}$ can become an important complementary tool for elucidation of structural properties inaccessible to other techniques and the development of molecular-functional devices.

\section{Acknowledgments}

This work was supported by the Engineering and Physical Sciences Research Council (EP/L002140/1). We acknowledge the Paul Scherrer Institut, Viligen, Switzerland for the provision of synchrotron radiation beam time at the PEARL beam line of the SLS and would like to thank Patrick Ascher for his support. The authors gratefully acknowledge the computing resources provided by the STFC Scientific Computing Department's SCARF cluster. PTPR was supported by the Advanced Characterisation of Materials (ACM) CDT.

\section{Data availability statement}

The data that support the findings of this study are openly available via https://zenodo.org at [28].

\section{ORCID iDs}

Paula L Lalaguna (D) https://orcid.org/0000-0003-2541-0826 Holly Hedgeland (D) https://orcid.org/0000-0003-3703-7942 Matthias K Muntwiler (D) https://orcid.org/0000-0002-66283977

Steven R Schofield (D) https://orcid.org/0000-0002-0727-3015 David A Duncan (D) https://orcid.org/0000-0002-0827-2022

\section{References}

[1] Xiang D, Wang X, Jia C, Lee T and Guo X 2016 Molecularscale electronics: from concept to function Chem. Rev $\mathbf{1 1 6}$ 4318-440
[2] Yates J T 1998 A new opportunity in silicon-based microelectronics Science 279 335-6

[3] Aviram A and Ratner M A 1974 Molecular rectifiers Chem. Phys. Lett. 29 277-83

[4] Aragones A C, Darwish N, Ciampi S, Sanz F, Gooding J J and Diez-Perez I 2017 Single-molecule electrical contacts on silicon electrodes under ambient conditions Nat. Commun. 8 8

[5] Hersam M C, Guisinger N P and Lyding J W 2000 Silicon-based molecular nanotechnology Nanotechnology 11 70-6

[6] Rakshit T, Liang G-C, Ghosh A W and Datta S 2004 Siliconbased molecular electronics Nano Lett. 4 1803-7

[7] Avasthi S, Qi Y, Vertelov G K, Schwartz J, Kahn A and Sturm J C 2011 Electronic structure and band alignment of 9,10phenanthrenequinone passivated silicon surfaces Surf. Sci. $\mathbf{6 0 5} 1308-12$

[8] Xiang D, Sydoruk V, Vitusevich S, Petrychuk M V, Offenhausser A, Kochelap V A, Belyaev A E and Mayer D 2015 Noise characterization of metal-single molecule contacts Appl. Phys. Lett. 1064

[9] Chadi D J 1979 Atomic and electronic structures of reconstructed Si(100) surfaces Phys. Rev. Lett. 43 43-7

[10] Wolkow R A 1992 Direct observation of an increase in buckled dimers on $\mathrm{Si}(001)$ at low temperature Phys. Rev. Lett. 68 $2636-9$

[11] Over H, Wasserfall J, Ranke W, Ambiatello C, Sawitzki R, Wolf D and Moritz W 1997 Surface atomic geometry of Si(001)$(2 \times 1)$ : a low-energy electron-diffraction structure analysis Phys. Rev. B 55 4731-6

[12] Carbone $M$ and Larsson K 2005 Methanol adsorption on the Si(100)- $2 \times 1$ surface: a first-principles calculation J. Phys.: Condens. Matter. 17 1289-300

[13] Schofield S R et al 2006 Phosphine dissociation and diffusion on $\mathrm{Si}(001)$ observed at the atomic scale J. Phys. Chem. B 110 $3173-9$

[14] Konecny R and Doren D J 1997 Adsorption of $\mathrm{BH}_{3}$ on $\mathrm{Si}(100)-$ $(2 \times 1)$ J. Phys. Chem. B 101 10983-5

[15] Warschkow O, Gao I, Schofield S R, Belcher D R, Radny M W, Saraireh S A and Smith P V 2009 Acetone on silicon (001): ambiphilic molecule meets ambiphilic surface Phys. Chem. Chem. Phys. 11 2747-59

[16] Schofield S R, Warschkow O, Belcher D R, Rahnejat K A, Radny M W and Smith P V 2013 Phenyl attachment to $\mathrm{Si}(001)$ via STM manipulation of acetophenone J. Phys. Chem. C 117 5736-41

[17] Schofield S R, Saraireh S A, Smith P V, Radny M W and King B V 2007 Organic bonding to silicon via a carbonyl group: new insights from atomic-scale images J. Am. Chem. Soc. 129 $11402-7$

[18] O'Donnell K M, Warschkow O, Suleman A, Fahy A, Thomsen L and Schofield S R 2015 Manipulating the orientation of an organic adsorbate on silicon: a NEXAFS study of acetophenone on $\operatorname{Si}(001)$ J. Phys.: Condens. Matter. 279

[19] Hossain M Z, Kato H S and Kawai M 2008 Self-directed chain reaction by small ketones with the dangling bond site on the $\mathrm{Si}(100)-(2 \times 1)-\mathrm{H}$ surface: acetophenone, a unique example J. Am. Chem. Soc. 130 11518-23

[20] Stöhr J 1996 NEXAFS Spectroscopy (Heidelberg: Springer)

[21] Woodruff D 2007 Adsorbate structure determination using photoelectron diffraction: methods and applications Surf. Sci. Rep. 62 1-38

[22] Muntwiler M et al 2017 Surface science at the PEARL beamline of the Swiss light source J. Synchrotron. Radiat. 24 354-66

[23] Duncan D A, Choi J I J and Woodruff D P 2012 Global search algorithms in surface structure determination using photoelectron diffraction Surf. Sci. $606278-84$ 
[24] Frisch M J et al 2013 Gaussian 09, Revision D.01 (Wallingford, CT: Gaussian, Inc.)

[25] Becke A D 1993 Density-functional thermochemistry. III. The role of exact exchange J. Chem. Phys. 98 $5648-52$

[26] Lee C, Yang W and Parr R G 1988 Development of the Colle-Salvetti correlation-energy formula into a functional of the electron density Phys. Rev. B 37 785-9
[27] Grimme S, Antony J, Ehrlich S and Krieg H 2010 A consistent and accurate $a b$ initio parametrization of density functional dispersion correction (DFT-D) for the 94 elements $\mathrm{H}-\mathrm{Pu} J$. Chem. Phys. 13219

[28] Lalaguna P L et al 2021 Data depository for the Determination of the preferred reaction pathway of acetophenone on $\mathrm{Si}(001)$ using photoelectron diffraction https://doi.org/10.5281/ zenodo.4549772 